\title{
Zweite Annäherung
}

Ein weiterer wichtiger Aspekt unserer Annäherung an die Romantik, ohne den wir - so glaube ich - dieses Phänomen nicht adäquat verstehen können, betrifft das neue Verhältnis zwischen Raum und Zeit und damit eine neue epistemologische Basis des Denkens im und für das 19. Jahrhundert. Die Grundlagen des Denkens, die Basis jener Denkstrukturen, in die wir neue Wissensbestände jeweils einlagern, haben sich im letzten Drittel des 18. Jahrhunderts so signifikant verändert, dass dadurch auch völlig neue Wissenskonstellationen entstehen konnten. Doch was ist mit der Episteme, was ist mit den Grundlagen des Denkens, was ist mit dem unserem Denken Selbstverständlichen, das sich verändert hat, gemeint?

Derartige Prozesse sind uns heute keineswegs neu, blicken wir nur einmal auf die zurückliegenden fünfzig Jahre unserer Geschichte zurück oder die Jahre seit Einführung des Personal Computers. Sie zogen nicht nur eine Auswirkung auf Darstellungsformen und Ästhetik der Präsentation - selbst auf der Ebene studentischer Hausarbeiten - nach sich, sondern bahnten auch neue Formen des relationalen Denkens in Zusammenhängen und des Arbeitens in Netzwerken an. Veränderungen in den Grundlagen des Denkens sind also gewiss nicht unabhängig von technologischen Fortschritten, sind aber auch nicht immer direkt auf solche beziehbar. Es gibt keine bessere Quelle als Literatur und Wissenschaft, um solche Transformationen präzise nachzuvollziehen. Vielleicht könnten wir das an der Schwelle zum 19. Jahrhundert entstehende neue Verhältnis zwischen Raum und Zeit am besten - wenn auch etwas allzu reduzierend - auf das Schlagwort „Verzeitlichung“ bringen. Was wäre dabei genauer unter einer solchen Temporalisierung aller wissenschaftlichen Denksysteme zu verstehen, die sich im Übergang vom 18. zum 19. Jahrhundert bemerkbar machte?

Der Soziologe und Kulturkritiker Wolf Lepenies, der frühere Direktor des Wissenschaftskollegs zu Berlin, hat in einer wichtigen Arbeit über das Ende der Naturgeschichte, die freilich Michel Foucault ganz zentrale - und viel zu selten signalisierte - Einsichten schuldet, vom Ende der Naturgeschichte gesprochen und mit diesem von ihm konstatierten Ende die Veränderung wissenschaftlicher Selbstverständlichkeiten am Ausgang des 18. Jahrhunderts korreliert. Ich bin davon überzeugt, dass es ohne diese grundlegende Veränderung der Selbstverständlichkeiten des Denkens gar keine Romantik gegeben hätte: Sie wäre ganz einfach nicht denkbar gewesen und hätte nicht stattgefunden. 


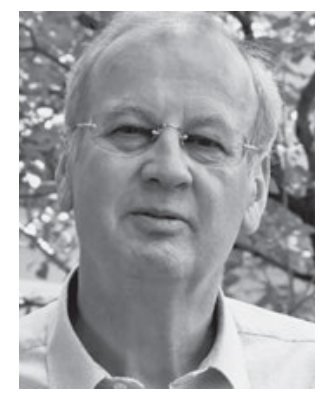

Abb. 6: Wolf Lepenies (Deuthen bei Allenstein, Ostpreußen, 1941).

Was ist aber mit dieser Veränderung, welche die Grundlagen für das Denken der Romantik legte, genau gemeint? Lassen wir hier am besten Wolf Lepenies selbst zu Wort kommen:

Dass in der zweiten Hälfte des 18. Jahrhunderts der Erfahrungsdruck die Naturgeschichte zur Temporalisierung ihrer Grundannahmen zwingt, bedeutet noch keine Vorwegnahme evolutionären Denkens. Darin drückt sich zunächst die Krise der Naturgeschichte aus, für deren Heftigkeit gerade die Zweideutigkeit der Versuche spricht, mit der man sie zu lösen versucht. Diese Zweideutigkeit kennzeichnet das Werk Lamarcks, sie ist bei Cuvier und Agassiz spürbar. Auf der einen Seite soll Lamarck den endgültigen Übergang zu einer Wissenschaft vom Leben vollziehen, auf der anderen Seite soll die Philosophie zoologique (1809) den Endpunkt einer Entwicklung markieren, die mit Linnés Systema naturae (1735) ihren Ausgangspunkt nimmt. Auf der einen Seite betrachtet Buffon die Flore française - deren drei Bände er in der königlichen Buchdruckerei drucken ließ - als Unterstützung seines Kampfes gegen Linné und die „Systeme“ und läßt Lamarck zum „botaniste du roi“ ernennen, auf der anderen sieht Etienne Geoffroy Saint-Hilaire in ihm den französischen Linné. [...]

In seiner Lettre sur le progrès des sciences hatte Maupertuis bei der Nachricht von einer „espèce mitoyenne“ zwischen den Affen und den Menschen, „behaarten Wilden, die Schwänze trugen“, ausgerufen: „Ich möchte lieber mit ihnen, als mit dem größten Schöngeist Europas eine Stunde im Gespräch verbringen.“ Mit dem Verzeitlichungsschub des späten 18. Jahrhunderts veraltet diese Form der humanen Neugier, die für die Naturgeschichte charakteristisch gewesen war. Nunmehr wird es möglich, die Wilden zu integrieren, die nicht länger in ihrer prinzipiellen Andersheit gedacht werden müssen, da ihre Gesellschaften als Stadien eines umfassenderen welthistorischen Prozesses erscheinen. Was an den Gesellschaften der Primitiven früher als Absurditäten aufgefaßt wurde, wird jetzt zu den Überbleibseln einer früheren Entwicklungsstufe. Nicht mehr der Rückgriff auf eine natürliche und universelle Vernunft, sondern eine Geschichtstheorie bestimmt nunmehr die Stellung des Abendlandes, das als das einzigartige Resultat dieses Entwicklungsprozesses erscheint, zu den Wilden. ${ }^{1}$

1 Lepenies, Wolf: Das Ende der Naturgeschichte. Wandel kultureller Selbstverständlichkeiten in den Wissenschaften des 18. und 19. Jahrhunderts. München: Carl Hanser 1978, S. 75 ff. 
In dieser ausführlich zitierten Passage wird ein grundlegender Übergang am Ausgang des 18. Jahrhunderts in den verschiedensten Wissenschaften vom Menschen und von der Natur angesprochen, der etwas verkürzt als ein Übergang vom Raum des statischen Tableaus zum beweglichen Raum der Zeit angesehen werden darf. Die enorme Akkumulation von Wissen und Wissensbeständen über die ganze Welt im Verlauf des 18. Jahrhunderts - denken Sie dabei nur an die Ergebnisse von Expeditionen und Reiseberichten ${ }^{2}$ und deren Sammlung und Archivierung! - lässt die räumlichen Tableaus, wie sie das System des Botanikers Linné charakterisierten, schlicht aus allen Nähten platzen. Was waren die wissenschaftstheoretischen Konsequenzen?

Unterschiedlichste Pflanzen und organische Lebewesen, die zuvor einfach verschiedenen Spezies und Arten zugeordnet waren und zwischen denen es nur Beziehungen der Ähnlichkeit, aber keine historischen Entwicklungsprozesse gab, werden nun in historischen Entwicklungsreihen angeordnet. Dadurch erscheinen plötzlich allerorten Prozessualitäten: Die eine Form ist historisch aus einer anderen Form entstanden, welche als früher diagnostiziert wird. Diese Transformationen des Denkens gelten nicht allein für die Phänomene der Natur, sondern auch für das Leben des Menschen.

Denn das Auftauchen einer „mittleren Gattung“ zwischen Affe und Mensch führte für Maupertuis, den ersten Präsidenten der Preußischen Akademie der Wissenschaften, nicht zu einer Überlegung im evolutionären Sinne, sondern nur zur Einfügung eines Zwischenbereichs zwischen zwei Kästchen des Tableaus, also zwischen Affe und Mensch. Immer stärker aber setzte sich ein neues Anordnungsschema durch, ein Schema der Verzeitlichung, in dem nun die unterschiedlichsten Lebewesen zumindest tendenziell in eine evolutionäre, zunächst aber erst einmal eine zeitliche Achse gebracht werden, welche ihren Entwicklungsgang angibt. Sie sehen, wie grundlegend sich die Möglichkeiten des Denkens verändern! Denn nun kommt eine Prozessualität der Befunde, eine Interpretation auf historisierender, verzeitlichender Ebene hinzu, die alte Markierungen und Denkgrundlagen zunächst transformiert und dann zunehmend verdrängt. Alle Phänomene der Natur wie des Menschen erscheinen plötzlich in einer neuen Dimensionalität: jener der Zeit.

Damit wird natürlich auch die strenge und strikte Trennung zwischen dem ,Eigenen' und dem ,Anderen‘ brüchig, könnte es doch nunmehr möglich werden, die unterschiedlichen organischen Lebensformen in einen Ablaufprozess zu

2 Nicht umsonst liegt ein Schwerpunkt der Untersuchung von Resieberichten im 18. Jahrhundert auch in der Vorlesung von Ette, Ottmar: ReiseSchreiben. Potsdamer Vorlesungen zur Reiseliteratur. Berlin - Boston: Walter de Gruyter 2020. 
stellen, der sozusagen die Grenzen zwischen Affen und Menschen im Sinne des 18. Jahrhunderts unterspült. Genau dies ist das Argument von Wolf Lepenies in der oben zitierten Passage zum Ende der Naturgeschichte. Denn letztere war, ihres Namens zum Trotz, keine Entwicklungsgeschichte in einem modernen Sinne. Wir können sie uns eher als etwas Geschichtetes vorstellen, wobei durch die einzelnen akkumulierten Schichten dieser Geschichte kein Zeitpfeil läuft.

Wenn Sie nun denken, diese gerade auch naturwissenschaftlichen Entwicklungen würden keine größere Rolle für Literatur und Kunst spielen, so täuschen Sie sich gewaltig; aber ich weiß ja: Sie würden sich hüten, so etwas zu denken und die Rolle der Wissenschaften so gering zu veranschlagen! Denn diese wissenschaftlichen Denkmodelle und Selbstverständlichkeiten gehen in die Grundlagen des Denkens der Menschen und damit auch der Literatur ein, die stets eine Sammlung verschiedenster Wissensbestände ist.

Daher kommt es nicht von ungefähr, wenn der große französische Romancier Honoré de Balzac im Vorwort zu seiner Comédie humaine gerade die Naturwissenschaften - ganz wie später im französischen Naturalismus Emile Zola eine vergleichbare Wendung vollziehen sollte - zum Ausgangspunkt seiner Romane macht und just Lamarck und insbesondere Geoffroy Saint-Hilaire einen wichtigen epistemologischen Platz in der Konzeption seines gewaltigen Romanzyklus zuweist. Balzac hatte verstanden, welch enorme Bedeutung der Veränderung wissenschaftlicher Diskurssysteme für die sprachliche Gemeinschaft und das Denken in einer bestimmten Kultur - in diesem Falle der abendländischen zukommt. Denn letztlich handelte es sich bei Zolas Menschlicher Komödie um eine Art Zoologie und Botanik, um eine zoologische Philosophie der Menschen zumindest seines französischen Lebensbereichs. Die Entwicklung neuer Denkmöglichkeiten führte aber notwendig zur Entwicklung neuer Schreibmöglichkeiten und darüber hinaus zu neuen Formen der Wahrnehmung durch unsere Sinne: Wir sehen anders, hören anders, schmecken anders, tasten anders, weil wir unsere Sinneseindrücke anderen Erfahrungsschemata und Erwartungshorizonten anpassen. Denn es wäre grundfalsch, würden wir die Sinneseindrücke sagen wir eines Menschen des Mittelalters oder der Aufklärung beim Benutzen von Salz, Pfeffer oder Zucker - mit den unsrigen im 21. Jahrhundert gleichsetzen.

Der französische Philosoph und Wissenschaftstheoretiker Michel Foucault hat diese Fragestellungen lange Jahre vor Wolf Lepenies bereits in grundlegender Weise in seinem 1966 erschienenen Buch Les mots et les choses, eingedeutscht Die Ordnung der Dinge, dargestellt. Es wäre nun ein abendfüllendes Programm, wollten wir dieses Buch, das sicherlich zu den wichtigsten Studien der zweiten Hälfte des 20. Jahrhunderts zählt und unsere Sichtweise auf fundamentale Veränderungen in der Geschichte unseres Denkens und unserer Diskursivitäten entscheidend geprägt hat, hier in aller Breite darstellen und analysieren. Aber ziehen 
wir zumindest einige Erkenntnisse aus diesem überaus reichen Band, um sie für unsere Vorlesung in Bezug auf zumindest zwei Polaritäten erkennbar und fruchtbar werden zu lassen!

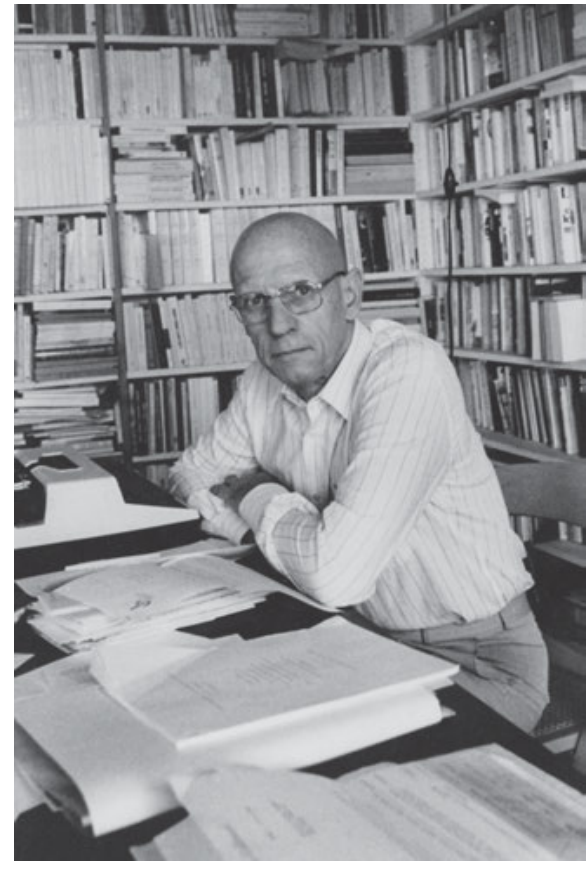

Abb. 7: Michel Foucault

(Poitiers, 1926 - Paris, 1984).

Zunächst zum Phänomen der Geschichte, von dem wir bereits gesehen haben, dass Geschichte nicht gleich Geschichte ist. Michel Foucault, der im Juni 1984 an den Folgen einer AIDS-Erkrankung verstarb und tragischerweise zu den frühen AIDS-Toten gehört - eine Pandemie, welche den Beginn der vierten Phase beschleunigter Globalisierung so eröffnete, wie die Coronavirus-Pandemie diese Phase endgültig abschloss -, hat im Grunde mit dem Ausgang des 18. und dem Übergang zum 19. Jahrhundert eine andere Episteme beginnen lassen. Dort verortete er den zeitlichen Übergang von einer Anfang des 17. Jahrhunderts entstandenen klassischen Episteme zu jener der Moderne.

Damit nun wuchs der Geschichte ein neuer Aufgabenbereich zu, der - so könnten wir aus heutiger Sicht sagen - ihr zum Teil in der zweiten Hälfte des vergangenen Jahrhunderts wieder abhandengekommen ist, weil andere Logiken und Denkmöglichkeiten aufgetreten sind, die nicht mehr notwendig von der Geschichte her ihre Wissensbestände zu ordnen gezwungen waren. Wir bezeichnen diese mittlerweile ebenfalls abgeschlossene Phase gemeinhin als Postmo- 
derne; doch da wir uns in der vergangenen Vorlesung ausführlich mit den mit ihr verbundenen Fragen beschäftigt haben, ${ }^{3}$ brauche ich an dieser Stelle nicht nochmals darauf einzugehen.

Nehmen wir aber einige Überlegungen Michel Foucaults zur Geschichte und zum Phänomen der Geschichtlichkeit auf, um besser zu verstehen, in welch grundlegender Weise sich eine neue Wissenskonstellation am Übergang zum 19. Jahrhundert herausbildete! Anhand des folgenden Zitats soll deutlich werden, dass die Verzeitlichung des Denkens nicht allein für die Naturwissenschaften und deren Wissenschaftsgeschichte, sondern auch für die Grundlagen des Denkens in einem allgemeinen Sinne gilt und daher gerade für die Literatur im langen 19. Jahrhundert relevant ist:

Die Geschichte bildet also für die Aufnahme der Humanwissenschaften ein gleichzeitig privilegiertes und gefährliches Gebiet. Für jede Wissenschaft vom Menschen bildet sie einen Hintergrund, der sie konstituiert und ihr ein Fundament und gewissermaßen eine Heimat festlegt. Sie bestimmt die kulturelle Fläche - die chronologische Episode, die geographische Einreihung -, wo man die Gültigkeit dieses Wissens erkennen kann. Aber sie kreist sie mit einer Grenze ein und zerstört von Anfang an ihren Anspruch, in dem Element der Universalität zu gelten. [...] Selbst wenn sie jeden Bezug auf die Geschichte vermeiden, tun die Humanwissenschaften (und dazu kann man in diesem Fall die Geschichte selbst zählen) nie etwas andres, als eine kulturelle Episode zu einer anderen in Beziehung zu setzen [...]. Jede Erkenntnis wurzelt in einem Leben, in einer Gesellschaft, einer Sprache, die eine Geschichte haben. Und in dieser Geschichte selbst findet sie das Element, das ihr gestattet, mit anderen Lebensformen, anderen Gesellschaftstypen und anderen Bedeutungen zu kommunizieren. ${ }^{4}$

In dieser sprachlich verdichteten Passage treten zwei unterschiedliche Begriffe deutlich hervor und miteinander in Beziehung: die Geschichte und das Leben. Sie ist, so macht Foucault sehr deutlich, zur Grundlage des Denkens in den Humanwissenschaften und letztlich des Denkens überhaupt geworden - und zwar gerade für den Zeitraum, der uns in unserer Vorlesung interessiert. Die Geschichte wird so könnten wir mit Blick auch auf die erste Fragestellung unserer dreigestaltigen Annäherung sagen - zu jenem Ort, an dem Universalität und Partikularität miteinander vermittelt werden. Geschichte entfaltet als Prozess gleichsam ihre eigene Geschichte, ohne in Selbstreflexivität zurückzufallen. Erst vor einem der-

3 Vgl. hierzu die literarästhetischen Überlegungen in Ette, Ottmar: Von den historischen Avantgarden bis nach der Postmoderne, S. 399-422 u. S. 609-613.

4 Foucault, Michel: Les mots et les choses. Paris: Gallimard 1966 [Originalzitat im Anhang]; hier Foucault, Michel: Die Ordnung der Dinge. Eine Archäologie der Humanwissenschaften. Frankfurt a.M.: Suhrkamp 1971, S. $444 \mathrm{ff}$. 
artigen Hintergrund wird es auch möglich, die Offenheit der Geschichte zu rekonstruieren, also jene vergangene Zukunft in ihrer Potentialität besser zu begreifen, von welcher vorhin in meinen Ausführungen die Rede war. Denn Geschichte in ihrer Verlaufsform enthält nicht nur die schiere Faktizität, wieso es so gekommen ist, sondern auch stets die Möglichkeiten, wie es auch hätte kommen können. Und dies ist, mit Aristoteles gesprochen, just die Aufgabe und die Stärke der Dichtkunst, also der Literatur.

Denn Michel Foucault weist mit guten Gründen darauf hin, dass es die Geschichte ist, die das Denken ermöglicht, zugleich aber auch bestimmte Grenzen einführt, die gerade auch das Partikulare, das einzelne Phänomen betreffen, das in seiner Potentialität nun greifbar und begreifbar wird. An dieser Stelle ergibt sich die Möglichkeit, die eine Episode mit einer anderen zu verknüpfen und daran wiederum das anzuschließen, was man mit Jean-François Lyotard in den Zeiten im Zeichen der Postmoderne - die zweifellos noch zur Moderne gehört - als „große Erzählungen“, als „grands récits“ bezeichnete. ${ }^{5}$ Oberhalb oder über der Geschichte platzieren sich dann - auf ihr fußend, sie aber zugleich beherrschend - jene Geschichtsphilosophien oder geschichtlich ausgerichteten Philosophien, die uns die Welt erklären und diese Weltepisoden, diese Weltgeschichten in eine einzige teleologische, also zielgerichtete Weltgeschichte umdeuten.

Ich würde mich hüten, diese Welterzählungen mit Jorge Luis Borges als große Fiktionen zu bezeichnen. Aber hierin liegt der Grund dafür, dass das 19. Jahrhundert so überaus reich war an Diskursivitätsbegründern, das heißt an Denkern, welche - wie Karl Marx oder Sigmund Freud - Erzählungen entwarfen, welche die einzelnen Erzählungen der Geschichte ,aufsammelten` und in eine kohärente Welterklärung aufnahmen. Im 19. Jahrhundert wurden die Grundlagen für so fundamentale und zum Teil bis heute wirksame Diskursivitäten gelegt wie diejenigen des Nationalismus oder Marxismus, des Kolonialismus oder des Rassismus, des Liberalismus oder Positivismus. Für all diese ,Geschichten‘ bildet die Entfaltung eines modernen, verzeitlichten und zielgerichteten Geschichtsbegriffs die unverzichtbare Grundlage.

Denn die gigantischen Geschichtsentwürfe, wie sie Hegel oder Marx im 19. Jahrhundert ausarbeiteten, wären ohne ein modernes Verständnis des Geschichtsbegriffs nicht möglich gewesen, gerade auch weil sie in ihrer Immensität und Totalität von einer deutlich geringeren Widersprüchlichkeit geprägt waren als der romaneske Entwurf etwa der Comédie humaine eines Honoré de Balzac. Denn Literatur baut ihre Entwürfe auf einer fundamentalen Polysemie,

5 Vgl. hierzu die klassische Studie von Lyotard, Jean-François: La condition postmoderne. Paris: Minuit 1979. 
einer strukturellen Vieldeutigkeit auf, welche bei der Konstruktion totalisierender und nicht selten auch totalitärer philosophischer Weltentwürfe gerade vermieden werden soll. Dies macht - wenn Sie mir diese Bemerkung gestatten - just den immensen Reichtum der Literaturen der Welt aus: nicht in einer totalitären Fiktion zu verharren, sondern die Vieldeutigkeit des Lebens selbst mit literarischen Mitteln darzustellen.

Damit inthronisiert sich im 19. Jahrhundert die Geschichte selbst als oberste Denk- und Gestaltungsmöglichkeit aller Geschichten, stammten sie aus den Naturoder den Humanwissenschaften. An diesem Punkt setzt jene Kunst ein, mit der wir uns in dieser Vorlesung beschäftigen wollen: die Kunst nämlich, Geschichten zu erzählen und Geschichten gut zu erzählen. Denn die moderne Konzeption von Geschichte ist auch die Grundlage des Geschichtenerzählens, und mehr noch: Sie ermöglicht eine bestimmte Art, Geschichten anzulegen und zu erzählen, wie sie gerade die Romantik entwickelt und zu einer ersten Blüte im 19. Jahrhundert geführt hat.

Doch Literatur ist keine Darstellung von Wirklichkeit, sondern die Darstellung gelebter Wirklichkeiten: Sie ist Mimesis in einem ebenso künstlerischen wie lebensbezogenen, lebenswissenschaftlichen Sinne. Es kommt also - wie im obigen Zitat von Foucault - an ganz zentraler Stelle das Leben in seiner Vielgestaltigkeit, seiner Vieldeutigkeit, seiner Vielverbundenheit hinzu. Dies ist ein grundlegender Aspekt, der oft in der Literaturwissenschaft zu kurz kommt, in unserer Vorlesung aber stark berücksichtigt werden soll.

Denn das Lebenswissen, Erlebenswissen, Überlebenswissen und Zusammenlebenswissen, ${ }^{6}$ das die Literaturen der Welt entfalten, gibt uns in und mit der Literatur die schier unendlichen Möglichkeiten an die Hand, die Lebensformen wie die Lebensnormen unterschiedlichster Zeiten, Regionen und Kulturen mitund nacherleben zu dürfen in einer Simulation des Lebens, welche nicht selten das reale Leben selbst gestaltet. In unserer Vorlesung über LiebeLesen ${ }^{7}$ haben wir gesehen, wie diese Fiktionen unsere Realitäten prägen und wie wir damit beginnen, Liebe zu lesen, zu erlernen, zu erleben und zu leben. Diese vieldimensionale und viellogische Eigenschaft von Literatur wollen wir auch in dieser Vorlesung nicht nur kennenlernen, sondern literarisch und ästhetisch genießen.

6 Vgl. hierzu ausführlich die Trilogie von Ette, Ottmar: ÜberLebenswissen. Die Aufgabe der Philologie. Berlin: Kulturverlag Kadmos 2004; ZwischenWeltenSchreiben. Literaturen ohne festen Wohnsitz (ÜberLebenswissen II). Berlin: Kulturverlag Kadmos 2005; sowie ZusammenLebensWissen. List, Last und Lust literarischer Konvivenz im globalen Maßstab (ÜberLebenswissen III). Berlin: Kulturverlag Kadmos 2010.

7 Vgl. Ette, Ottmar: LiebeLesen, S. 3-32 u. S. 720-726. 